\title{
Heuristics Core Mapping in On-Chip Networks for Parallel Stream-Based Applications
}

\author{
Piotr Dziurzanski and Tomasz Maka \\ Szczecin University of Technology, \\ ul. Zolnierska 49, 71-210 Szczecin, Poland \\ \{pdziurzanski, tmaka\}@wi.ps.pl
}

\begin{abstract}
A novel approach for implementation of multimedia streaming applications into mesh Networks on Chips structure is proposed. We provide a new multi-path routing algorithm together with heuristic algorithms for core mapping so that minimize the total transfer in the hardware implementation. The proposed approach has been tested with two popular stream-based video decompression algorithms. The experimental results confirming the proposed approach are provided.
\end{abstract}

Keywords: Multimedia streaming applications, On-Chip routing algorithm, IP core mapping, Multi-path routing.

\section{Introduction}

Computational-intensive multimedia applications are especially well suited for parallel and distributed processing due to their data-dominated algorithms that can be split intro a number of stages. These stages can be implemented in separate computational units working in a pipeline-like way and transmitting each other streams of relatively large, but usually fixed, amount of data. Some widelyknown examples of the algorithms of that type are, e.g., MPEG-4, DAB, DVB, and many others. In these applications, it is usually required to keep an assumed quality level of service and meet real-time constraints [7.

Multi Processor Systems on Chips (MPSoCs) are often considered as suitable hardware implementations of these applications [3]. As each processing unit of a MPSoC can realize a single stage of streaming application processing, it is still problematic to connect these units together. The simplest point to point $(\mathrm{P} 2 \mathrm{P})$ connections require too much space, whereas bus-based connections result in large number of conflicts and, consequently, despite various arbitrage techniques decrease the overall performance of the whole system [4. Besides, both P2P and bus-based realizations do not scale well with the constantly increasing number of independent Intellectual Property (IP) cores (i.e., computational units) required by contemporary devices dealing with a number of various algorithms in a single system [1].

In order to omit these obstacles, the packet-based Network-on-Chip (NoC) paradigm for the designs of chips realizing distributed computation has been introduced [2]. The recent popularity of this approach can be attributed to a

M. Bubak et al. (Eds.): ICCS 2008, Part I, LNCS 5101, pp. 427-435, 2008.

(C) Springer-Verlag Berlin Heidelberg 2008 
lower number of conflicts in a chip with a large number of cores. It is reported that NoC architectures offer high bandwidth and good concurrent communication capability, but they require additional mechanisms to overcome problems typical for packet switching communication, such as packet deadlock or starvation, but the techniques known for traditional computer networks have to be altered before applying to on-chip networks [6. A mesh is one of the most often used on-chip network topologies owing to its regularity and reliability due to the existence of many redundant interconnections between nodes. In NoCs, each mesh node is comprised of the IP core realizing a particular stage of the algorithm and a router which is typically connected to four neighboring nodes.

A typical NoC implementation utilizes packet switching approach that is called wormhole routing [5. In this technique, each packet is split into smaller units of equal length, flits (flow control units). Usually, the first flits contain some routing information, such as the destination address. Having obtained the routing information, a wormhole router selects the next-hop router and establishes the path to that neighboring router. This path can be used exclusively for transferring the current package flit by flit as long as the whole package has not been transferred. The next-hop router typically does not store the whole package in its buffers, but tries to establish a connection with another router being selected for the transfer. If another package is to be sent through the connection already used for transferring a package, its transfer is deferred as long as all flits of the previous package has not been sent. This situation is known as contention and may result in significant decreasing of efficiency. Contentions are especially likely to be observed in various data-intensive applications, where large streams of data are transferred in every second. This may result in violating the real-time constraints and thus making an MPSoC designing in this way inappropriate for the task.

The most popular routing algorithm used in NoCs, named XY, can be also viewed as inappropriate for switching large streams of information. According to this algorithm, a flit is firstly routed according to the $\mathrm{X}$ axis as long as the $\mathrm{X}$ coordinate is not equal to the $\mathrm{X}$ coordinate of the destination core, and then the flit is routed vertically. Despite being deadlock-free [5], this algorithm is not adaptive and thus is not equipped with mechanism for decreasing the contention level.

Taking into consideration the above mentioned facts, it follows that in order to design a NoC-based MPSoC for multimedia streaming applications it is necessary to (i) propose a routing algorithm that is more suitable to this task that the traditional XY algorithm and (ii) to propose a mapping scheme of IP cores into mesh nodes that decreases the contention level.

\section{Proposed Design Flow}

The first stage of the proposed approach is to construct a flow network for a given stream-based algorithm. The processing blocks of the algorithm are identified and the transfers between them are computed. We describe all transfers in a 
Table 1. Transfers table for H.264 decoder

\begin{tabular}{|c|c|c|}
\hline Source & Destination & Transfer [bps] \\
\hline \hline 1 & 2 & 11744051 \\
\hline 2 & 3 & 503316480 \\
\hline 3 & 4 & 503316480 \\
\hline 4 & 5 & 788529152 \\
\hline 5 & 8 & 788529152 \\
\hline 1 & 5 & 360710144 \\
\hline 1 & 6 & 37748736 \\
\hline 6 & 4 & 11744051 \\
\hline 1 & 7 & 251658240 \\
\hline 7 & 4 & 1560281088 \\
\hline 8 & 7 & 2348810240 \\
\hline
\end{tabular}

so-called transfer table. An example of the transfer table for the H.264 decoder is presented in Table 11. The numbers in the Source and Destination columns represent indices of the nodes in the flow network.

Then, we have to determine the mapping of the cores into the mesh structure leading to the improved performance of the NoC structure.

The impact of the mapping on the final implementation properties is very significant in case of the traditional wormhole XY routing approach. For the example of MPEG-4 decoder [9], the difference between the required capacity and the best and the worst mappings is about 203.04 per cent.

For example, the XY algorithm applied to the H.264 video decoder 8 for core permutation 0-2-3, 7-8-4, 1-6-5 in the first, second, and the third row, respectively, leads to the transfers presented in Fig. 1.

In this situation, the maximal transfer between adjacent cores is relatively high being equal to $2240 \mathrm{Mbit} / \mathrm{s}$. It means that in every second such amount of data is to be transferred between cores 8 and 7, so that the NoC infrastructure

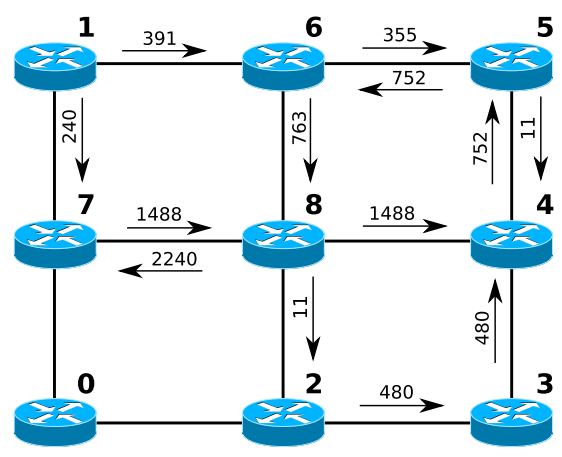

Fig. 1. Transfers between cores for the H.264 decoder, XY routing algorithm (in Mbit/s) 
has to offer capacities large enough to cope with this transfer. Assuming the most popular regular NoC mesh architecture, all the links have to have equal capacities, so all of them have to be capable of transferring $2240 \mathrm{Mbit} / \mathrm{s}$. However, the majority of the remaining links are utilized in small percentage of this maximal value. It may be expressed with the standard deviation value, which is equal to about 598.36 Mbit/s. Thus, we assumed that the standard deviation express the transfer balancing level. The smaller is the standard deviation, the transfers are closer to each other. Moreover, only 13 links out of 24 are utilized, which results in unbalanced transfers and poor utilization of the available resources.

This is our main motivation to introduce a routing scheme we named tapeworm routing. An efficiency of this routing algorithm depends on a structure of cores and their connections to each other, which is defined with a mapping of the flow network nodes functionality into NoC cores.

Having selected an appropriate mapping, it is important to balance transfers between each path in a NoC structure. Our mapping algorithms need on its input a complete list of data transfers in the network flow built at the previous stage. The proposed technique takes advantage of the well-known Ford-Fulkerson method for determining maximal throughput of the network between a set of cores.

The example of the three successive steps of the tapeworm algorithm is presented in Fig. 2. In this figure, the numbers written above links mean a flow and a remaining available capacity. The data length to be sent between cores $\mathrm{S}$ and $\mathrm{D}$ is equal to 70 bits. At the first stage, 30 bits of the link between routers 2 and 3 have been already allocated. As the available capacity between routers 1 and 2 (i.e., the link selected by the XY rule) is only 50 bits, 50 bits are sent by this link, and the remaining 20 bits are sent by the alternative route to the 4 th router. In router 2 , the package is further segmented: 20 bits follow the path to the router 3 , whereas 30 bits are sent to router 5 . Thus, the total data length sent between routers 5 and 6 is equal to 50 bits.

A few algorithms for heuristic core mapping into a NoC structure are provided in the following section.

\section{On-Chip Cores Mapping Heuristics}

In order to determine the appropriate cores mapping into $\mathrm{NoC}$ nodes, i.e., the mapping leading to the minimal value of transfers between cores while using the tapeworm algorithm, it is possible to use an exact algorithm. However, its application is reasonable to the size of the NoC mesh lower than $4 \mathrm{x} 4$ cores due to its immense computational complexity $O\left(n ! \cdot n^{2}\right)$, where $n$ is the number of cores. For larger $n$, however, it is possible to use heuristic algorithms that do not significant worsen the final result, as it is shown in the sequel of this paper. Below, we propose three heuristics that according to experimental results (presented in Section 4), lead to results close to the exact approach.

We start our algorithm with a generating of a population of random core permutations to be mapped into the NoC structure. Then, we execute one of the provided below heuristics (Fig. 3. 3 ) for every permutation a number of times. 


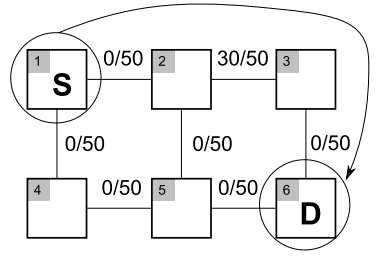

(a)

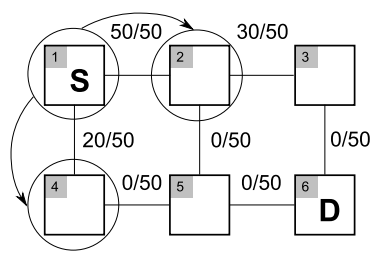

(b)

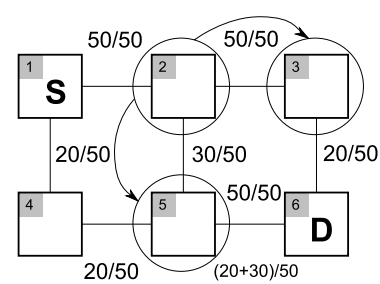

(c)

Fig. 2. Successive steps of the tapeworm algorithm

1. src $\leftarrow$ select randomly a core $c_{1}$

2. do

3. $\quad$ nDir $\leftarrow$ random direction (Left, Right, Up, Down)

4. while (move toward nDir direction is possible)

5 . dest $\leftarrow$ select the adjacent core of $c_{1}, c_{2}$, in the nDir direction

6. if (the exchange between src and dest cores decreases the total transfer)

7. $\quad \operatorname{swap}\left(c_{1}, c_{2}\right)$

Fig. 3. Pseudo-code of heuristics 1 for cores mapping

1. select randomly an item from NoC transfers table between src and dest

2. do

3. $\quad$ nDir $\leftarrow$ random direction (Left, Right, Up, Down)

4. while (move dest toward nDir direction is possible)

5 . dest $\leftarrow$ select the adjacent core of dest in the nDir direction

6. if (the exchange between src and dest cores decreases the total transfer)

7. $\quad$ swap (src, dest)

Fig. 4. Pseudo-code of heuristics 2 for cores mapping

1. select randomly an item from NoC transfers table between src and dest

2. $d_{0} \leftarrow$ calculate Manhattan distance between scr and dest cores

4. $\left(d_{1}, d_{2}, d_{3}, d_{4}\right) \leftarrow$ calculate Manhattan distance between src and all neighbors of dest

5. if $\left(\min \left(d_{0}, d_{1}, d_{2}, d_{3}, d_{4}\right) \neq d_{0}\right)$

6. $n_{\min } \leftarrow$ neighbor with the lowest distance

7. swap (dest, $n_{\min }$ )

Fig. 5. Pseudo-code of heuristics 3 for cores mapping

We decided to terminate when a maximum number of generations has been produced, or the obtained transfers do not decrease for a specific number of steps. As we tested our approach for multimedia applications split into relatively low number of cores (9), we could compare the obtained results with the exact solutions. 
In the first heuristics (Fig. 3), we select randomly two neighboring cores, and then compute total number of bits transmitted in a second for two cases: (i) for the existing core mapping (ii) and the one obtained after the exchange of the two selected cores. If the latter approach is characterized with a lower transfer, the exchange is performed.

In the second approach (Fig. 4), we select randomly a single transfer from the transfer table of the algorithm to be mapped. Next, the direction from the set \{left, right, up, down\} is selected, and a new mapping, when the destination core is exchanged with the core situated directly on the selected side is performed. Similarly to the previous heuristic, both total transfers before and after the exchange are computed and if the core with the second one is lower, the exchange is carried out.

In the third heuristics (Fig. [5), we also select a single transfer from the transfer table. Then, we calculate the Manhattan distance between the source and the destination cores of the selected transfer. The Manhattan distances between the source and all the neighbors of the destination core are also computed and, if the distance is lower for any of the neighbors, it is exchanged with the destination core. In case when more than one neighbor is characterized with a lower distance, the lowest of them is chosen. It is worth stressing that in the case of this approach there is no need of computing the total transfer in the NoC, which is time-saving as presented in the next section.

\section{Experimental Results}

In order to verify the above provided approach, we have chosen the MPEG-4, and H.264 decoders and decided to execute all the heuristic described in the previous section for determining the permutation mapping that is characterized with the lowest total transfer in the NoC realization.

For the selected decoders, we have built flow networks and determined the amount of data transmitted between their nodes in every second. We executed an implementation of the proposed approaches 2000 times and concluded that in every case a local minimum has been found at a relatively early iteration (the solution was the same as obtained with the exact algorithm). For the two first approaches, the local minimum has been found no later than in 367th iteration, whereas for the 3rd approach it was no later than 1684th iteration. However, the vast majority of the found local minima has been found much earlier, what is

Table 2. Comparison of the mean transfers and standard deviations obtained with the proposed approaches (in Mbit/s)

\begin{tabular}{|c||c|c|c|c|c|c|}
\cline { 2 - 7 } \multicolumn{1}{c|}{} & \multicolumn{2}{c|}{ Approach 1 } & \multicolumn{2}{c|}{ Approach 2 } & \multicolumn{2}{c|}{ Approach 3 } \\
\hline Algorithm & Mean & StdDev & Mean & StdDev & Mean & StdDev \\
\hline MPEG-4 & 1734.74 & 9.03 & 1734.63 & 9.1 & 1726.75 & 1.96 \\
\hline H.264 & 828.32 & 2.79 & 828.39 & 2.64 & 829.21 & 2.64 \\
\hline
\end{tabular}




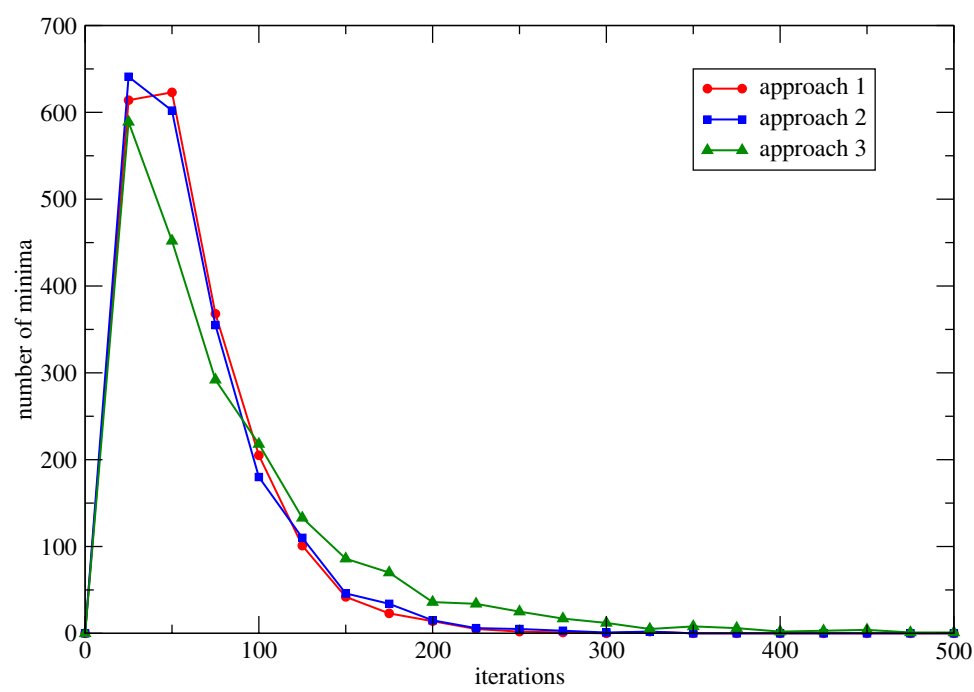

Fig. 6. Minima histogram for H.264

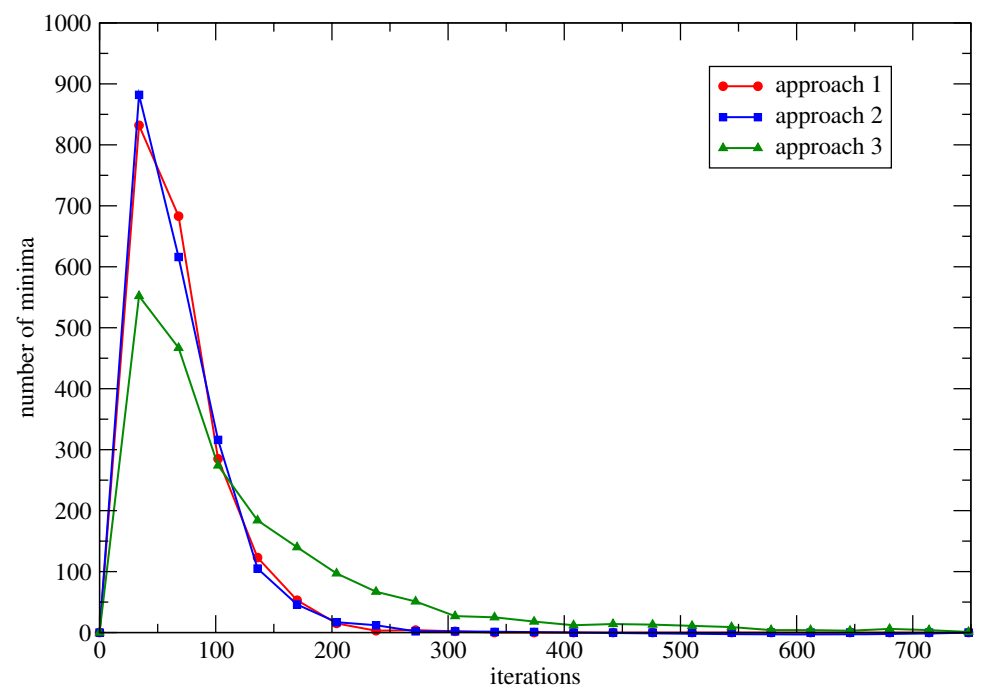

Fig. 7. Minima histogram for MPEG-4

depicted by histograms presented in Fig. 6] and Fig. 7 for H.264 and MPEG-4, respectively. Only few instances has been found in further iterations than 300 .

In Tab. 2, we have presented average transfers and standard deviations for a set of 1000 algorithm executions. All three approaches resulted in values close 
to the minimum. For H.264 (MPEG-4), we obtained the global minimum for $59.55,54.9$, and $26.7(47.15,48.4$, and 96.85) per cent of cases for the first, second, and the third approach, respectively.

In the two first approaches, it is important to stress that for each iteration one has to determine the total transfer for a number of times. We have measured that for 200 iterations the transfer is to be determined 60100 times. As our tapeworm routing runs for 8.224 seconds in average (Intel Pentium 4 CPU 3 $\mathrm{GHz}, 1 \mathrm{~GB}$ RAM memory), the running time for the two first approaches is less than 6 days. On the other hand, the third approach produces results in $0.5 \mathrm{~s}$. Although the first time seems huge, especially in a comparison with the second one, it is still much less, the exact algorithm is practically unacceptable for even $4 \mathrm{x} 4$ cores (a few million years of computation). The execution time of the first two approaches depends only polynomially on the size of the mesh.

\section{Conclusion}

We described our approach for implementing data-intensive streaming multimedia applications in Network on Chip based on the mesh topology. We focused on two phases: mapping of algorithm stages into the target NoC's nodes and developing a new multi-path routing algorithm. Both our proposals benefit from the static streams of data transferred between IP cores being known at the design stage. We provided three heuristic mapping algorithms and compared them with the exact solutions. The provided experimental results, based on two reallife multimedia decoder examples, showed that in majority of cases our heuristics give results equal to the exact solution at relatively early iterations.

The computational complexity of the proposed heuristics is polynomial with respect to the number of cores, whereas the complexity of the exact solution is intractable as being estimated with $O\left(n ! \cdot n^{2}\right)$, where $n$ is the number of IP cores to be mapped. Consequently, the proposed technique allows us to practically solve the problem of mapping a streaming multimedia application into a NoCbased MPSoC in a reasonable time so that the data transfers between IP cores are balanced and close to the global minimum.

\section{References}

1. Bjerregaard, T., Mahadevan, S.: A Survey of Research and Practices of Networkon-Chip. ACM Computing Surveys (CSUR) 38, Article 1 (2006)

2. Dally, W.J., Towels, B.: Route Packets, Not Wires: On-Chip Interconnection Networks. In: 38th ACM IEEE Design Automation Conference (DAC), pp. 684-689 (2001)

3. Kavaldjiev, N., et al.: Routing of guaranteed throughput traffic in a network-on-chip. Technical Report TR-CTIT-05-42 Centre for Telematics and Information Technology, University of Twente, Enschede (2005)

4. Lee, H.G., Chang, N., Ogras, U.Y., Marculescu, R.: On-chip communication architecture exploration: A quantitative evaluation of point-to-point, bus, and networkon-chip approaches. ACM Transactions on Design Automation of Electronic Systems 12(3), article no. 23 (2007) 
5. Li, M., Zeng, Q.A., Jone, W.B.: DyXY: a proximity congestion-aware deadlockfree dynamic routing method for network on chip. In: 43rd ACM IEEE Design Automation Conference (DAC), pp. 849-852 (2006)

6. Ogras, U.Y., Marculescu, R.: Prediction-based Flow Control for Network-on-Chip Traffic. In: 43rd ACM IEEE Design Automation Conference (DAC), pp. 839-844 (2006)

7. Smit, G.J.M., et al.: Efficient Architectures for Streaming DSP Applications, Dynamically Reconfigurable Architectures. Internationales Begegnungs- und Forschungszentrum fuer Informatik (IBFI), Schloss Dagstuhl, Germany (2006)

8. van der Tol, E.B., Jaspers, E.G.T., Gelderblom, R.H.: Mapping of H.264 decoding on a multiprocessor architecture. In: Image and Video Communications and Processing, Santa Clara, CA, USA, vol. 5022, pp. 707-718 (January 2003)

9. van der Tol, E.B., Jaspers, E.G.T.: Mapping of MPEG-4 Decoding on a Flexible Architecture Platform. In: Media Processors 2002, San Jose, CA, USA, vol. 4674, pp. 362-363 (January 2002) 